\title{
Mapping the convergent temporal epileptic network in left and right
}

\section{temporal lobe epilepsy}

Peng Fang ${ }^{\mathrm{a}^{*}}$, Jie An ${ }^{\mathrm{b} *}$, Ling-Li Zeng ${ }^{\mathrm{a}}$, Hui Shen ${ }^{\mathrm{a}}$, Shijun Qiu ${ }^{\mathrm{b}}$, Dewen $\mathrm{Hu}^{\mathrm{a}}$

a College of Mechatronics and Automation, National University of Defense Technology, Changsha, Hunan 410073, China

${ }^{\text {b }}$ Department of Medical Imaging, The First Affiliated Hospital of Guangzhou University of Chinese Medicine, Guangzhou, Guangdong 510405, China

* The authors contributed equally to the manuscript.

Corresponding author:

Dewen Hu, Address: College of Mechatronics and Automation, National University of Defense Technology, Changsha, Hunan 410073, China, E-mail: dwhu@nudt.edu.cn

Shijun Qiu, Address: Department of Medical Imaging, The First Affiliated Hospital of Guangzhou University of Chinese Medicine, Guangzhou, Guangdong 510405, China, E-mail: qiu-sj@163.com 


\section{Abstract}

Left and right mesial temporal lobe epilepsy (mTLE) with hippocampal sclerosis (HS) exhibits similar functional and clinical dysfunctions, such as depressive mood and emotional dysregulation, implying that the left and right mTLE may share a common network substrate. However, the convergent anatomical network disruption between the left and right HS remains largely uncharacterized. This study aimed to investigate whether the left and right mTLE share a similar anatomical network.

We examined 43 (22 left, 21 right) mTLE patients with HS and 39 healthy controls using diffusion tensor imaging. Machine learning approaches were applied to extract the abnormal anatomical connectivity patterns in both the left and right mTLE.

The left and right mTLE showed that 28 discriminating connections were exactly the same when compared to the controls. The same 28 connections showed high discriminating power in comparisons of the left mTLE versus controls (91.7\%) and the right mTLE versus controls (90.0\%); however, these connections failed to discriminate the left from the right mTLE. These discriminating connections, which were diminished both in the left and right mTLE, were primarily located in the limbic-frontal network, partially agreeing with the limbic-frontal dysregulation model of depression.

These findings suggest that left and right mTLE share a convergent circuit, which may account for the mood and emotional deficits in mTLE and may suggest the neuropathological mechanisms underlying the comorbidity of depression and mTLE.

Key words: temporal lobe epilepsy; temporal epileptic network; diffusion tensor imaging; anatomical connection; machine learning approaches 
Abbreviations: $\mathrm{MRI}=$ magnetic resonance imaging; $\mathrm{TLE}=$ temporal lobe epilepsy; $\mathrm{HS}=$ hippocampal sclerosis; DTI = diffusion tensor imaging; LOOCV = leave-one-out crossvalidation; $\mathrm{ROI}=$ region of interest; $\mathrm{SVM}=$ support vector machine; $\mathrm{GR}=$ generalization rate; $\mathrm{SC}=$ specificity $\mathrm{SS}=$ sensitivity $; \mathrm{ROC}=$ receiver operating characteristic $; \mathrm{FA}=$ fractional anisotropy. dlPFC $=$ prefrontal cortex; $\mathrm{OFC}=$ orbitofrontal cortex 


\section{Introduction}

Mesial temporal lobe epilepsy (mTLE) with hippocampal sclerosis (HS), which is often associated with cognitive impairment [1], has been considered as a focal disease centered on a lateralized focus for a long time [2]. However, previous magnetic resonance imaging (MRI) studies have demonstrated widespread abnormalities in various cortical regions and networks [3$6]$, suggesting that mTLE is a brain disease that involves network dysfunction $[7,8]$.

Pioneering studies indicated that the left and right mTLE involved distinct underlying pathological and etiological substrates [9]. In our last study, we found that the left mTLE could be distinguished from the right mTLE. However, the left mTLE partly exhibited a comparable connectivity pattern to the right mTLE [10]. Furthermore, similar functional connectivity reductions were found in both left and right mTLE [11]. Left mTLE patients even exhibit similar clinical dysfunctions to right mTLE patients, such as depressive mood, emotional dysregulation, memory deficits [12] and even major depression [13]. More importantly, some studies failed to detect significant white matter differences in direct comparisons between left and right mTLE [14]. The neuropathology underlying these phenomena remains unclear. One hypothesis is that white matter abnormalities reflect a secondary effect of ongoing seizure activity, representing downstream axonal degeneration [15], such that left and right mTLE may share a common extratemporal network disruption.

In the current study, we investigated whether left and right mTLE share a common disrupted anatomical network; to address this question, we aimed to characterize the convergent disruptions of the anatomical networks in left and right mTLE using machine learning approaches. Whereas mass-univariate methods consider each individual variable separately, machine learning approaches take into account patterns of information that may be presented across multiple variables [16]. Thus, machine learning approaches may provide increased sensitivity for extracting stable patterns from neuroimaging data and for detecting subtle and spatially distributed differences in the brain [17]. First, we performed diffusion tensor imaging (DTI) probabilistic tractography to extract whole-brain anatomical networks. Then, machine learning approaches were used to extract the most discriminating connections and to investigate the convergent anatomical network disruptions between left and right mTLE. 


\section{Methods}

This study was approved by the Research Ethics Review Board of the Institute of Mental Health of Southern Medical University. Each participant was informed of the details of the project, and written informed consent was obtained from all participants in accord with the standards of the Declaration of Helsinki. We confirmed that all potential participants who declined to participate or otherwise did not participate were eligible for treatment (if applicable) and were not disadvantaged in any way by not participating in this study.

\subsection{Participants}

We enrolled 43 consecutive right-handed patients suffering from unilateral HS and mTLE who received a presurgical evaluation at the Guangdong 999 Brain Hospital. The diagnosis and lateralization of the seizure focus to the left mTLE $(n=22)$ or the right mTLE $(n=21)$ were determined based on a comprehensive evaluation, including a detailed history, video-EEG telemetry and neuroimaging. An increase in the T2 fluid-attenuated inverted recovery signal in the hippocampus was used as the diagnostic criterion for HS, and the site of HS was concordant with the epileptogenic site in all patients. None of the patients had a mass lesion (including tumor, vascular malformation or malformations of cortical development) or suffered from traumatic brain injury or a psychiatric disorder, but all patients experienced degeneralized seizures. After MRI acquisition, all patients received anterior temporal lobectomy. Following qualitative histopathological analysis, HS was detected in all patients. Thirty-nine age-, genderand education-matched right-handed healthy control participants were recruited for this study. The demographic and clinical data are displayed in Table 1.

\subsection{Imaging protocol}

All participants were scanned using a 1.5 T Philips Intera MR scanner. During scanning, foam pads were used to reduce head motion and scanner noise. Diffusion-weighted images were obtained using a single-shot echo-planar imaging sequence according to the following parameters: repetition time $(\mathrm{TR})=11,000 \mathrm{~ms}$; echo time $(\mathrm{TE})=71.6 \mathrm{~ms}$; field of view $(\mathrm{FOV})=$ $230 \mathrm{~mm} \times 230 \mathrm{~mm}$; matrix size $=144 \times 144$; voxel dimensions $=1.6 \times 1.6 \times 2 \mathrm{~mm}$; slice thickness $=2 \mathrm{~mm} ; 32$ non-collinear diffusion directions with a b-value of $800 \mathrm{~s} / \mathrm{mm}^{2}$ and one additional volume without diffusion weighting $\left(b=0 \mathrm{~s} / \mathrm{mm}^{2}\right)$; and 73 transverse slices without gaps, 
covering the entire brain. We also acquired high-resolution 3D brain anatomical images using a T1-weighted MP-RAGE sequence according to the following parameters: $\mathrm{TR}=25 \mathrm{~ms}, \mathrm{TE}=4.6$ $\mathrm{ms}, \mathrm{FOV}=240 \mathrm{~mm} \times 240 \mathrm{~mm}$, matrix size $=256 \times 256$, and 140 contiguous axial slices with slice thickness $=1 \mathrm{~mm}$.

\subsection{DTI data processing}

Diffusion tensor images were corrected for distortions caused by head motion and eddy currents using affine registration in Eddy Current Correction [18]. Then, the resulting images were brain extracted using the Brain Extraction Tool [19], and a diffusion tensor model was fit to each voxel using DTIFit to generate images of FA and other parameters. Here, we adopted an automated anatomical labeling (AAL) parcellation method to parcellate the cortex into 116 regions of interest (ROIs) [20]. Then, the local probability distribution of the fiber directions was estimated for each voxel using BedpostX. We adopted ProbtrackX for probabilistic tractography, which tracked fibers between each pair of ROIs by sampling 5000 streamline fibers per voxel using a turning threshold of 60 degrees. The ROI associated with node $v$ is denoted as $\operatorname{ROI}(v)$. If $\operatorname{ROI}(v)$ contained $n$ voxels, the total number of fibers connecting to $R O I(v)$ was $5000 \times n$. Given the number of fibers from $R O I(v)$ to $R O I(u)$ was $m$, the connections between the nodes $R O I(v)$ and $R O I(u)$ were defined as edge $e(v, u)=\frac{m}{5000 \times n}$. The connectivity strength between $R O I(v)$ and $R O I(u)$ was defined as $E(v, u)=\frac{\mathrm{e}(\mathrm{v}, \mathrm{u})+\mathrm{e}(\mathrm{u}, \mathrm{v})}{2}$ [21]

\subsection{Feature selection and classification}

We applied a two-sample $t$-test to identify the connections that were significantly different between groups, which were the most discriminating features. Then, we adopted a locally linear embedding algorithm (LLE) to reduce the feature space dimensionality to a more manageable level. Finally, a support vector machine (SVM) with the default Gaussian radial basis function kernel was applied for classification. Here, we performed two classifications of left mTLE versus controls and right mTLE versus controls with whole brain connections. We extracted exactly the same features from these two classifications as the convergent features. Two-way group classifications using the convergent features of the left mTLE, right mTLE and controls were performed to assess these features. 
Because the sample size was limited in this study, we adopted a leave-one-outcross-validation (LOOCV) strategy to estimate the generalization rate $(G R)$ of the SVM classifier [17]. In each fold of LOOCV, one subject was extracted as the test group, and the other N-1 subjects were retained to train the SVM classifier. First, the most discriminating features were selected from the N-1 training subjects using TSTT and further projected into the feature space, in which variabilities between patients and controls were best represented. Then, training samples were used to train the classifier and test samples were employed to evaluate the classifier performance by comparing classification results with the ground truth class labels. As there are N samples, LOOCV trained the classifier $\mathrm{N}$ times. The performance of each classifier was quantified for its Sensitivity (SS), Specificity (SC) and Generalization Rate (GR) based on the results of LOOCV. The SS indicated the proportion of patients correctly classified, and the SC represented the proportion of controls that were correctly classified. The overall proportion of samples correctly classified was represented by GR. We applied permutation tests and receiver operating characteristic (ROC) curves to assess the statistical significance of the observed classification accuracy values.

\section{Results}

\subsection{Whole-brain classification}

Using the LOOCV strategy, the SVM classifier achieved 93.4\% accuracy for the left mTLE versus controls and $90.0 \%$ accuracy for the right mTLE versus controls (Table 2). Because the training data differed for each LOOCV, the selected features varied slightly in each LOOCV. However, 97 and 94 discriminating features, referred to as the consensus features [22], were detected in every LOOCV for the left mTLE versus controls and for the right mTLE versus controls, respectively. These two sets of consensus features were considered the most discriminating features in the classification. All of the consensus connections were diminished in both the left and right mTLE compared to the controls.

The left and right mTLE patients exhibited a similarly diminished connectivity pattern compared to controls (displayed in Figure 1 and shown in Table S1 and Table S2) and 28 (30\%) disconnections overlapped between the two group discriminating connections. These shared disconnections in the left and right mTLE were primarily located in the limbic-frontal and temporo-occipital network (displayed in Figure 2 and shown in Table S3). Little connections 
were inter-hemispheric, most of the connections were primarily intra-hemispheric.

\subsection{Analysis with convergent connections}

For classifications performed on convergent connections with a two-way group comparison strategy, the classification of the left mTLE versus controls, the right mTLE versus controls and the left mTLE versus the right mTLE resulted in accuracies of $90.2 \%, 90.0 \%$ and $69.7 \%$, respectively (Table 2). The permutation test and ROC curves demonstrated that both the left and right mTLE were significantly different from controls (Figure S1, Figure S2 and Figure S3). However, the left mTLE did not show significant differences with the right mTLE according to the permutation test and ROC curves (Figure S2 and Figure S3).

\section{Discussion}

In this study, we adopted a probabilistic diffusion tracking method and machine learning approaches to investigate the convergent connectivity patterns between the left and right mTLE. The left mTLE partly displayed convergent abnormal connectivity patterns with the right mTLE. These shared discriminating connections were primarily located in the limbic-frontal and temporo-occipital subsystems. Our results suggest that left and right mTLE share a convergent dysregulated anatomical network, in contrast to a single dysfunctional structure localized to the focus-lateralized temporal lobe.

\subsection{Classification}

As pattern analysis based on machine learning approaches are capable of extracting stable patterns from neuroimaging data, finding significant neuroimaging-based biomarkers and identifying patients from control participants at individual subject level [17, 23], machine learning approaches are increasingly adopted in neuroscience image studies in recent years[2225]. In the present study, TSTT and LLE algorithm were used to extract feature connections, while SVM was adopted for classification. identify altered anatomical connectivity in depressed patients compared with controls. Being effective and simple, TSTT and SVM had been widely used in neuroimaging studies $[22,26]$. LLE not only aims at reducing data dimensionality but also attempts to discover an intrinsic low-dimensional structure of the data[17]. In addition, permutation tests were employed to assess statistical significance of the classification results. Permutation tests validated the relationship between the data and the labels with a maximum 
probability of being wrong at 0.0001 . To assess statistical significance of the observed classification accuracy, permutation tests were applied using the GR as the statistic that measured dissimilarity between two populations [27]. The class labels of the training data were first randomly permuted, and then the cross-validation was carried out for each set of the labelpermuted data. The entire permutation process was repeated 10,000 times [24]. Given the null hypothesis that the classifier could not learn the relationship between the data and the labels reliably when the generalization rate obtained by the classifier trained on the real class labels was lower than the $95 \%$ confidence interval of the classifier trained on randomly relabeled class labels. For any value of the estimated generalization rate $\mathrm{GR}_{0}$, the appropriate $\mathrm{p}$-value $\mathrm{P}\left(\mathrm{GR}_{0}\right)$ represented the probability of observing a classification prediction rate which was no less than $\underline{G R}_{0}$. We were supposed to reject the null hypothesis and declare that the classifier learned the relationship between the data and the labels with a probability of being wrong at most $\mathrm{P}\left(\mathrm{GR}_{0}\right)$.

In the present study, 93.4\% of the left mTLE were differentiated from the controls, and $90.0 \%$ of the right mTLE were identified from the controls. The results from permutation tests and ROC curves also demonstrated the efficiency of the classifier. We adopted the same discriminating connections from these two classifications for classifications of left mTLE versus controls, right mTLE versus controls and left mTLE versus right mTLE. The identifications from mTLE to controls achieved promising accuracy, which were $90.2 \%$ for left mTLE versus controls and $90.0 \%$ for right mTLE versus controls. However, the classification accuracy of the left mTLE and right mTLE was as low as $69.7 \%$. Furthermore, the permutation test and ROC curve did not show significant differences between the left and right mTLE. These results suggested that the same discriminating connections can be viewed as convergent dysfunctional connections between the left and right mTLE.

\subsection{Altered convergent anatomical networks in mTLE}

\section{Limbic-frontal network}

Altered connectivity was found in both mTLE groups in the limbic-frontal network with regions that are known to be involved in mood and emotional processing [28], such as the dorsolateral prefrontal cortex (dlPFC), the orbitofrontal cortex (OFC), and the cingulate cortex [29]. The frontal lobe network represents a major route of seizure propagation from the mesial temporal focus, and impaired frontal-lobe emotional and cognitive functions have been reported in TLE 
[30]. Patients with epilepsy frequently accompany with mood disorders [31]. Depression is reported as the principal psychiatric deficit in TLE $[32,33]$. The frequency of depression even takes up 50-60\% of TLE patients [34]. Furthermore, mTLE patients have a lifetime prevalence of any type of mood disorder compared with controls or patients with any other disorders [35]. The $\underline{\text { PFC and precuneus, showed large region weight in the result, have been considered to play a }}$ central role in the default mode network (DMN). The altered connections were partly overlapped with the DMN, which was in line with DMN dysfunction of depression [36] and may account for the cognitive decline in TLE patients [37]. Dysregulated connections in the limbic-frontal network of mTLE patients were also consistent with limbic-frontal dysregulation model of depression involving negative mood and emotional processing [24, 38]. Studies even show limbic-frontal network dysfunction is highly associated with depressive mood in TLE [39, 40]. The OFC, the insula, the dlPFC, and the cingulate cortex are primarily implicated in subclinical depressive mood, and the observed abnormalities are consistent with prior studies of major depression [31]. The anterior cingulate cortex, which is strongly involved in the pathophysiology of depression, has been increasingly viewed as an indispensable region in anatomical and functional models of mood regulation and emotional processing [41]. The dysfunctional connections between the PFC, the OFC, the cingulate cortex, the insula, and the basal ganglia are located in the DMN, dorsal-ventral system and an affective fronto-limbic circuitry, which are involved in depressive mood and emotional regulation $[36,42,43]$. Together with the precentral gyrus, these areas play important roles in the limbic-cortical dysregulation model of depression involving depressive mood and emotional regulations [38], suggesting a neuropathological mechanism that accounts for the mood and emotional deficits both in the left and right mTLE.

\section{Temporo-occipital network}

Abnormalities in anatomical connectivity were found in the temporo-occipital network in both left and right mTLE, involving connections between the fusiform, the inferior, middle and superior temporal gyri, and visual cortical areas such as cuneus, lingual, inferior and middle occipital gyri. Studies show that the severity or duration of depression in TLE is correlated with temporal lobe dysfunction [44]. Dysfunctional temporo-occipital connections were overlapping with the inferior longitudinal fasciculus and may contribute to visual memory dysfunction in mTLE [45]. Visual cortices together with ventral visual projections to the temporal cortices are involved in mood modulation [43]. The temporo-occipital cortex is known to process facial 
emotional expressions and responses to fear [46]. Temporo-occipital white matter dysfunction was significantly associated with longer illness duration and earlier age of illness onset in bipolar depression [47]. Thus, it is reasonable to speculate that an aberrant temporo-occipital network may contribute to mood, emotion and memory dysfunction in mTLE patients.

\subsection{Advantages and limitations}

A growing body of MRI-based mTLE studies has focused on voxel-based analysis of focal abnormalities [48]. These studies provide valuable insight into the pathological mechanisms of TLE, but some issues should be noted. First, seed-based methods limit the obtained information to the selected ROIs and make it difficult to examine anatomical connectivity patterns on a whole-brain scale [49]. Second, traditional group-level statistical methods do not provide a mechanism for evaluating the discriminative power of the identified connections at the individual level [16]. This study took advantage of machine learning approaches and probabilistic tractography to investigate the convergent whole-brain anatomical network disruptions between left and right mTLE. We demonstrated, from a connectivity perspective, that the left and right mTLE share a 'depressive circuit' located in the limbic-frontal and temporo-occipital networks. On the other hand, there are several limitations that should be acknowledged. First, there was no clinical data or EEG data that could be used for correlation analyses in this study. We will perform psychological assessment to evaluate anxiety and depressive symptoms for all participants in the future. Second, the relatively small sample size reduced our confidence in the results. It would be desirable to utilize the method presented in this paper with larger samples. Third, machine learning approaches may lose some altered connections when we choose the most powerful features for analysis. Thus, we should assess the significance of the less powerful features in the future. Finally, we lack patients suffering from other types of epilepsy to further investigate whether this 'depressive circuit' exists in other types of epilepsy.

\section{Conclusions}

In conclusion, the present study is the first study to investigate the common anatomical network disturbances between left and right mTLE which had been long neglected in mTLE using machine learning approaches. The results showed that left and right mTLE patients could be differentiated from healthy controls with high classification accuracy, indicating that the most discriminating connections could be viewed as potential biomarkers for mTLE with HS. The 
most discriminating connections' strength was decreased in both left and right mTLE patients, and these connections were primarily located in the prefrontal-limbic network and temporooccipital network. These findings suggest that left and right mTLE share a convergent circuit, which may account for the mood and emotional deficits in mTLE and may suggest the neuropathological mechanisms underlying the comorbidity of depression and mTLE.

\section{Acknowledgements}

The authors thank Olaf Sporns from Indiana University Bloomington for his modification of this paper and constructive suggestions. This study was supported by the National Science Foundation of China (61503397, 61420106001, 61375111, 81271389, and 81471251). Peng Fang is the guarantor of this work and, as such, had full access to all the data in the study and takes responsibility for the integrity of the data and the accuracy of the data analysis. P.F. and J.A. wrote the manuscript and researched data. L.L.Z and H.S. reviewed/edited the manuscript. D.H. contributed to discussion and reviewed/edited manuscript. S.Q. researched data and contributed to discussion. All authors have approved the final article and declare that there are no conflicts of interest related to this study. . 


\section{References}

[1] B. Hermann, M. Seidenberg, J. Jones, The neurobehavioural comorbidities of epilepsy: can a natural history be developed?, Lancet Neurol 7 (2008) 151-160.

[2] M.R. Trenerry, C.R. Jack, R.J. Ivnik, F.W. Sharbrough, G.D. Cascino, K.A. Hirschorn, W.R. Marsh, P.J. Kelly, F.B. Meyer, MRI hippocampal volumes and memory function before and after temporal lobectomy, Neurology 43 (1993) 1800-1805.

[3] B.C. Bernhardt, N. Bernasconi, L. Concha, A. Bernasconi, Cortical thickness analysis in temporal lobe epilepsy: reproducibility and relation to outcome, Neurology 74 (2010) 1776-1784.

[4] S.S. Keller, N. Roberts, Voxel-based morphometry of temporal lobe epilepsy: an introduction and review of the literature, Epilepsia 49 (2008) 741-757.

[5] N.L. Voets, C.F. Beckmann, D.M. Cole, S. Hong, A. Bernasconi, N. Bernasconi, Structural substrates for resting network disruption in temporal lobe epilepsy, Brain 1 (2012) 1-8.

[6] B.C. Bernhardt, N. Bernasconi, H. Kim, A. Bernasconi, Mapping thalamocortical network pathology in temporal lobe epilepsy, Neurology 78 (2012) 129-136.

[7] F. Riederer, R. Lanzenberger, M. Kaya, D. Prayer, W. Serles, C. Baumgartner, Network atrophy in temporal lobe epilepsy: a voxel-based morphometry study, Neurology 71 (2008) 419-425.

[8] J. Engel, Jr, Mesial temporal lobe epilepsy: what have we learned?, Neuroscientist 7 (2001) 340-352.

[9] J. Janszky, F.G. Woermann, P. Barsi, R. Schulz, P. Halasz, A. Ebner, Right hippocampal sclerosis is more common than left after febrile seizures, Neurology 60 (2003) 12091210.

[10] P. Fang, J. An, L.-L. Zeng, H. Shen, F. Chen, W. Wang, S. Qiu, D. Hu, Multivariate pattern analysis reveals anatomical connectivity differences between the left and right mesial temporal lobe epilepsy, NeuroImage: Clinical 7 (2015) 555-561.

[11] G. Doucet, K. Osipowicz, A. Sharan, M.R. Sperling, J.I. Tracy, Extratemporal functional connectivity impairments at rest are related to memory performance in mesial temporal epilepsy, Hum Brain Mapp 34 (2013) 2202-2216.

[12] R. Coras, E. Pauli, J. Li, M. Schwarz, K. Rossler, M. Buchfelder, H. Hamer, H. Stefan, I. Blumcke, Differential influence of hippocampal subfields to memory formation: insights from patients with temporal lobe epilepsy, Brain 137 (2014) 1945-1957.

[13] K. Getz, B. Hermann, M. Seidenberg, B. Bell, C. Dow, J. Jones, A. Woodard, P. Rutecki, R. Sheth, D. O'Leary, V. Magnotta, Negative symptoms in temporal lobe epilepsy, Am J Psychiatry 159 (2002) 644-651.

[14] P. Besson, V. Dinkelacker, R. Valabregue, L. Thivard, X. Leclerc, M. Baulac, D. Sammler, O. Colliot, S. Lehéricy, S. Samson, S. Dupont, Structural connectivity differences in left and right temporal lobe epilepsy, NeuroImage 100 (2014) 135-144.

[15] D.W. Gross, Diffusion tensor imaging in temporal lobe epilepsy, Epilepsia 52 Suppl 4 (2011) 32-34.

[16] M. Oquendo, E. Baca-Garcia, A. Arte’s-Rodri'guez, F. Perez-Cruz, H. Galfalvy, H. Blasco-Fontecilla, D. Madigan, N. Duan, Machine learning and data mining: strategies for hypothesis generation, Mol Psychiatry (2012) 1-4.

[17] H. Shen, L. Wang, Y. Liu, D. Hu, Discriminative analysis of resting-state functional connectivity patterns of schizophrenia using low dimensional embedding of fMRI, 
NeuroImage 49 (2010) 3110-3121.

[18] J. Andersson, M. Jenkinson, S.M. Smith, Non-linear registration, aka Spatial normalisation, FMRIB technical report TR07JA2 (2007).

[19] S.M. Smith, Fast robust automated brain extraction, Human Brain Mapping 17(3) (2002) 143-155.

[20] N. Tzourio-Mazoyer, B. Landeau, D. Papathanassiou, F. Crivello, O. Etard, N. Delcroix, B. Mazoyer, M. Joliot, Automated anatomical labeling of activations in SPM using a macroscopic anatomical parcellation of the MNI MRI single-subject brain, NeuroImage 15 (2002) 273-289.

[21] P. Fang, L.-L. Zeng, H. Shen, L. Wang, B. Li, L. Liu, D. Hu, Increased cortical-limbic anatomical network connectivity in major depression revealed by diffusion tensor imaging, PLoS ONE 7 (2012) e45972.

[22] N.U. Dosenbach, B. Nardos, A.L. Cohen, D.A. Fair, J.D. Power, J.A. Church, S.M. Nelson, G.S. Wig, A.C. Vogel, C.N. Lessov-Schlaggar, K.A. Barnes, J.W. Dubis, E. Feczko, R.S. Coalson, J.R. Pruett, D.M. Barch, S.E. Petersen, B.L. Schlaggar, Prediction of individual brain maturity using fMRI, Science 329 (2010) 1358-1361.

[23] Y. Fan, R.E. Gur, R.C. Gur, X. Wu, D. Shen, M.E. Calkins, C. Davatzikos, Unaffected Family Members and Schizophrenia Patients Share Brain Structure Patterns: A HighDimensional Pattern Classification Study, Biol Psychiatry 63 (2008) 118-124.

[24] L.-L. Zeng, H. Shen, L. Liu, L. Wang, B. Li, P. Fang, Z. Zhou, Y. Li, D. Hu, Identifying major depression using whole-brain functional connectivity: a multivariate pattern analysis, Brain 135 (2012) 1498-1507.

[25] L.L. Zeng, H. Shen, L. Liu, D. Hu, Unsupervised classification of major depression using functional connectivity MRI, Human brain mapping 35 (2014) 1630-1641.

[26] S.G. Costafreda, C. Chu, J. Ashburner, C.H.Y. Fu, Prognostic and diagnostic potential of the structural neuroanatomy of depression, PLoS ONE 4 (2009) e6353.

[27] P. Golland, B. Fischl, Permutation tests for classification: towards statistical significance in image-based studies, Inf Process Med Imaging 2732 (2003) 330-341.

[28] K.N. Ochsner, J.J. Gross, The cognitive control of emotion, Trends Cogn Sci 9 (2005) 242-249.

[29] M. Ballmaier, A.W. Toga, R.E. Blanton, E.R. Sowell, H. Lavretsky, J. Peterson, Anterior cingulate, gyrus rectus, and orbitofrontal abnormalities in elderly depressed patients: an MRI-based parcellation of the prefrontal cortex, Am J Psychiatry 161 (2004) 99-108.

[30] S. Takaya, T. Hanakawa, K. Hashikawa, A. Ikeda, N. Sawamoto, T. Nagamine, K. Ishizu, H. Fukuyama, Prefrontal hypofunction in patients with intractable mesial temporal lobe epilepsy, Neurology 67 (2006) 1674-1676.

[31] A. Lothe, A. Didelot, A. Hammers, N. Costes, M. Saoud, F. Gilliam, P. Ryvlin, Comorbidity between temporal lobe epilepsy and depression: a [18F]MPPF PET study, Brain 131 (2008) 2765-2782.

[32] J.J. Lin, M. Mula, B.P. Hermann, Uncovering the neurobehavioural comorbidities of epilepsy over the lifespan, Lancet 380 ( 2012) 1180-1192.

[33] A.M. Kanner, Depression in epilepsy is much more than a reactive process, Epilepsy currents 3 (2003) 202.

[34] J.I. Victoroff, D. Benson, S.T. Grafton, J. Engel, Jr, J.C. Mazziotta, DEpression in complex partial seizures electroencephalography and cerebral metabolic correlates, Archives of Neurology 51 (1994) 155-163.

[35] K.D. Valente, G. Busatto Filho, Depression and temporal lobe epilepsy represent an epiphenomenon sharing similar neural networks: clinical and brain structural evidences, 
Arquivos de neuro-psiquiatria 71 (2013) 183-190.

[36] B. Li, L. Liu, K.J. Friston, H. Shen, L. Wang, L.-L. Zeng, D. Hu, A treatment-resistant default mode subnetwork in major depression, Biological psychiatry 74 (2013) 48-54.

[37] J.S. Damoiseaux, C.F. Beckmann, E.S. Arigita, F. Barkhof, P. Scheltens, C.J. Stam, S.M. Smith, S.A.R.B. Rombouts, Reduced resting-state brain activity in the "default network" in normal aging, Cerebral Cortex 18 (2008) 1856-1864.

[38] D.A. Seminowicz, H.S. Mayberg, A.R. McIntosh, K. Goldapple, S. Kennedy, Z. Segal, S. Rafi-Tari, Limbic-frontal circuitry in major depression: a path modeling metanalysis, NeuroImage 22 (2004) 409-418.

[39] N. Kemmotsu, N.E. Kucukboyaci, K.M. Leyden, C.E. Cheng, H.M. Girard, V.J. Iragui, E.S. Tecoma, C.R. McDonald, Frontolimbic brain networks predict depressive symptoms in temporal lobe epilepsy, Epilepsy research 108 (2014) 1554-1563.

[40] S. Chen, X. Wu, S. Lui, Q. Wu, Z. Yao, Q. Li, D. Liang, D. An, X. Zhang, J. Fang, Resting-state fMRI study of treatment-naive temporal lobe epilepsy patients with depressive symptoms, Neuroimage 60 (2012) 299-304.

[41] J. Brown, C.M. Cooper - Kuhn, G. Kempermann, H. Van Praag, J. Winkler, F.H. Gage, H.G. Kuhn, Enriched environment and physical activity stimulate hippocampal but not olfactory bulb neurogenesis, European Journal of Neuroscience 17 (2003) 2042-2046.

[42] M.L. Phillips, W.C. Drevets, S.L. Rauch, R. Lane, Neurobiology of emotion perception I: the neural basis of normal emotion perception, Biol Psychiatry 54 (2003) 504-514.

[43] R. Canbeyli, Sensorimotor modulation of mood and depression: an integrative review, Behavioural brain research 207 (2010) 249-264.

[44] B. Bell, J.J. Lin, M. Seidenberg, B. Hermann, The neurobiology of cognitive disorders in temporal lobe epilepsy, Nat Rev Neurol 7 (2011) 154-164.

[45] C. Elger, C. Helmstaedter, M. Kurthen, Chronic epilepsy and cognition, Lancet Neurol 3(11) (2004) 663-672.

[46] P. Krolak-Salmon, M.-A. Hénaff, A. Vighetto, O. Bertrand, F. Mauguière, Early amygdala reaction to fear spreading in occipital, temporal, and frontal cortex: A depth electrode ERP study in human, Neuron 42 (2004) 665-676.

[47] M.V. Zanetti, M.P. Jackowski, A. Versace, J.R. Almeida, S. Hassel, F.L. Duran, G.F. Busatto, D.J. Kupfer, M.L. Phillips, State-dependent microstructural white matter changes in bipolar I depression, European archives of psychiatry and clinical neuroscience 259 (2009) 316-328.

[48] L. Concha, C. Beaulieu, D.W. Gross, Bilateral limbic diffusion abnormalities in unilateral temporal lobe epilepsy, Ann Neurol 57 (2005) 188-196.

[49] M.P. van den Heuvel, H.E.H. Pol, Exploring the brain network: A review on resting-state fMRI functional connectivity, Eur Neuropsychopharmacol 20 (2010) 519-534. 


\section{TABLES}

Table 1. Demographic and clinical data.

\begin{tabular}{lllll}
\hline Variable & Left mTLE & Right mTLE & Control & $p$-value \\
\hline Sample size & 22 & 21 & 39 & \\
& & & & \\
Gender (M/F) & $14 / 8$ & $11 / 10$ & $22 / 17$ & $0.45^{\mathrm{a} *}$ \\
Age (median, range in years) & $26.2 \pm 7.4(18-42)$ & $28.33 \pm 7.8(18-43)$ & $26.11 \pm 7(18-44)$ & $0.97^{\mathrm{a} *}$ \\
Education (years) & $11.1 \pm 2$ & $11.5 \pm 2.3$ & $11.4 \pm 2.28$ & $0.82^{\mathrm{a} *}$ \\
Duration of episode (years) & $12.2 \pm 7$ & $12.9 \pm 7.4$ & & $0.74^{\mathrm{b} *}$ \\
& & & & $0.59^{\mathrm{b} *}$ \\
\hline
\end{tabular}

mTLE $=$ mesial temporal lobe epilepsy $; \mathrm{M}=$ male $; \mathrm{F}=$ female.

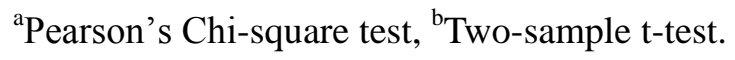

* No significant difference between groups (significance defined as $p<0.05$ ). 
Table 2. Comparison of classification performance using different features.

\begin{tabular}{|c|c|c|c|c|}
\hline \multirow{2}{*}{ Features } & \multicolumn{4}{|c|}{ Result } \\
\hline & $G R$ & $S S$ & $S C$ & $P T p$-value \\
\hline L mTLE vs Controls (whole brain) & $93.4 \%$ & $81.8 \%$ & $100.0 \%$ & $<0.0001$ \\
\hline R mTLE vs Controls (whole brain) & $90.0 \%$ & $81.8 \%$ & $94.9 \%$ & $<0.0001$ \\
\hline L mTLE vs Controls (28 connections) & $90.2 \%$ & $86.4 \%$ & $92.3 \%$ & $<0.0001$ \\
\hline R mTLE vs Controls (28 connections) & $90.0 \%$ & $85.7 \%$ & $92.3 \%$ & $<0.0001$ \\
\hline L mTLE vs R mTLE (28 connections) & $69.7 \%$ & $72.7 \%$ & $66.7 \%$ & 0.0548 \\
\hline
\end{tabular}

$G R=$ generalization rate; $S S=$ sensitivity $S C=$ specificity $; P T=$ permutation test L mTLE = left mesial temporal lobe epilepsy; R mTLE = right mesial temporal lobe epilepsy; vs = versus. 


\section{FIGURE LEGENDS}

Figure 1. Region weights and distribution of the most discriminating anatomical connections in classification for the left mTLE versus controls and the right mTLE versus controls. Top: classification for left mTLE versus controls. Bottom: classification for right mTLE versus controls. The consensus connections are displayed on a surface rendering of the brain. The thickness of the connections adjusts according to their connectivity strength. Green and orange connections represent decreased and increased connectivity in mTLE, respectively. The connections are all decreased in left and right mTLE compared to controls. The diameter of the sphere represents the corresponding region weight of the ROI. The ROIs are color-coded according to brain areas $($ Red $=$ frontal cortex; Orange $=$ limbic cortex; Yellow $=$ parietal cortex; Sky blue $=$ cerebellar cortex Deep blue $=$ temporal cortex Purple $=$ occipital cortex $) . \mathrm{R}=$ right hemisphere, $\mathrm{L}=$ left hemisphere med = medial; mid = middle; ope = opercular; tria = triangular; sup = superior; ORB = Orbital Frontal SFG = Superior Frontal ACG = Anterior Cingulum; SMA = Supplementary Motor Area; PreCG = Precentral; ROL = Rolandic INS = Insula; AMYG = Amygdala $;$ DCG = Middle Cingulate PCG = Post Cingulum $;$ PUT = Putamen HIP = Hippocampus; ITG = Inferior Temporal; MTG = Middle Temporal; STG = Superior Temporal; TPO = Temporal Pole; FFG = Fusiform CUN = Cuneus MOG = Middle occipital; PCUN = Precuneus; $\mathrm{PoCG}=$ Postcentral; Cer $=$ Cerebelum . 
Figure 2. Region weights and distribution of the convergent anatomical connections in the

left and right mTLE. The abbreviations and legends are as the same as that in Figure 1. 
L SMAL SMAR SFGdorL I 1 ( CO DEGR $Y$ CUNR SFGmed ACGR, ROLR PCG.L. ACG.L CAULOS MTG.L ACG.L PUT:L MTG.L
HIRIL ORBmedY AMYG. ITGLITGR Cer8.L

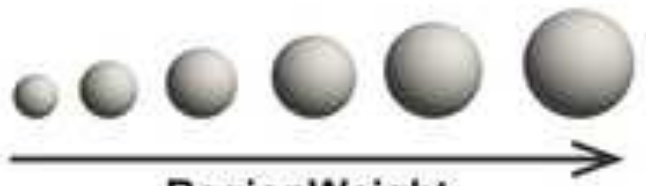

RegionWeight

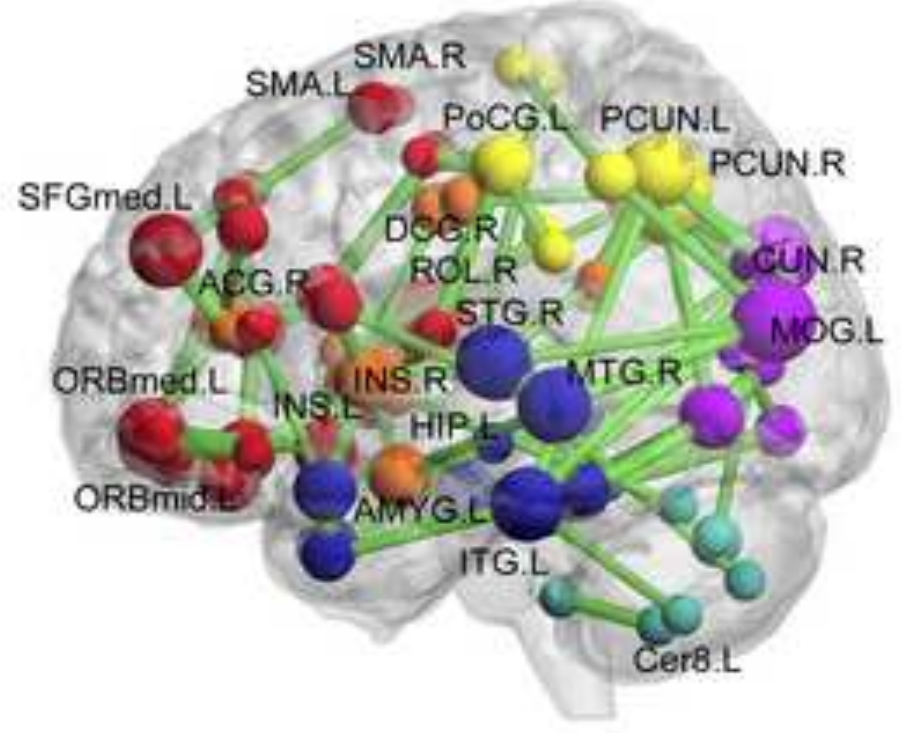

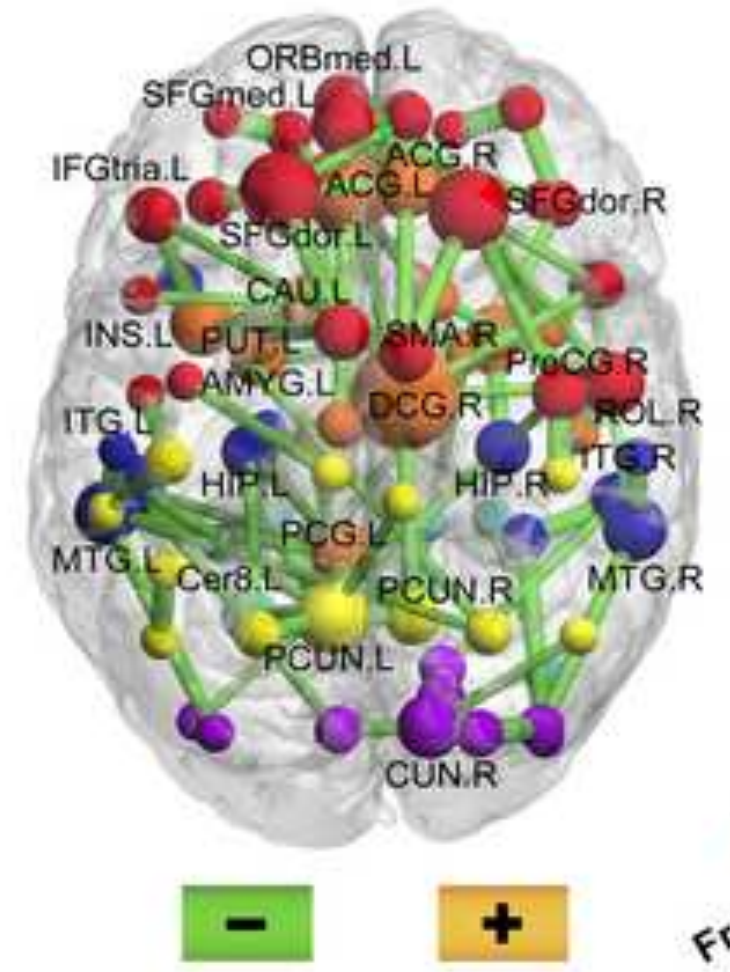

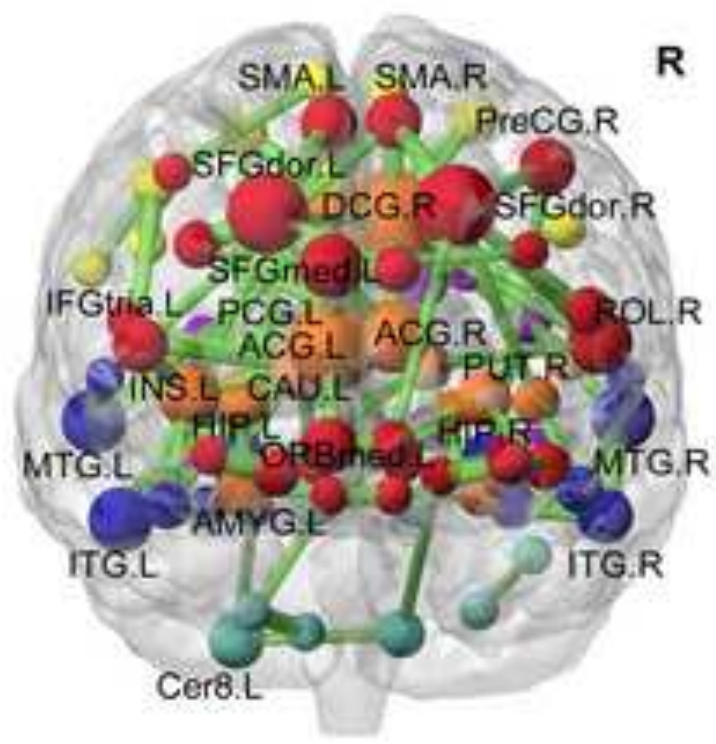

$\mathbf{R}$

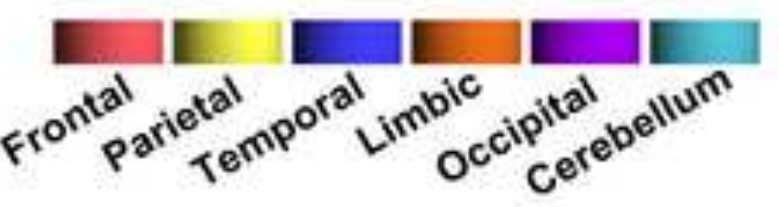

ORBrned.L
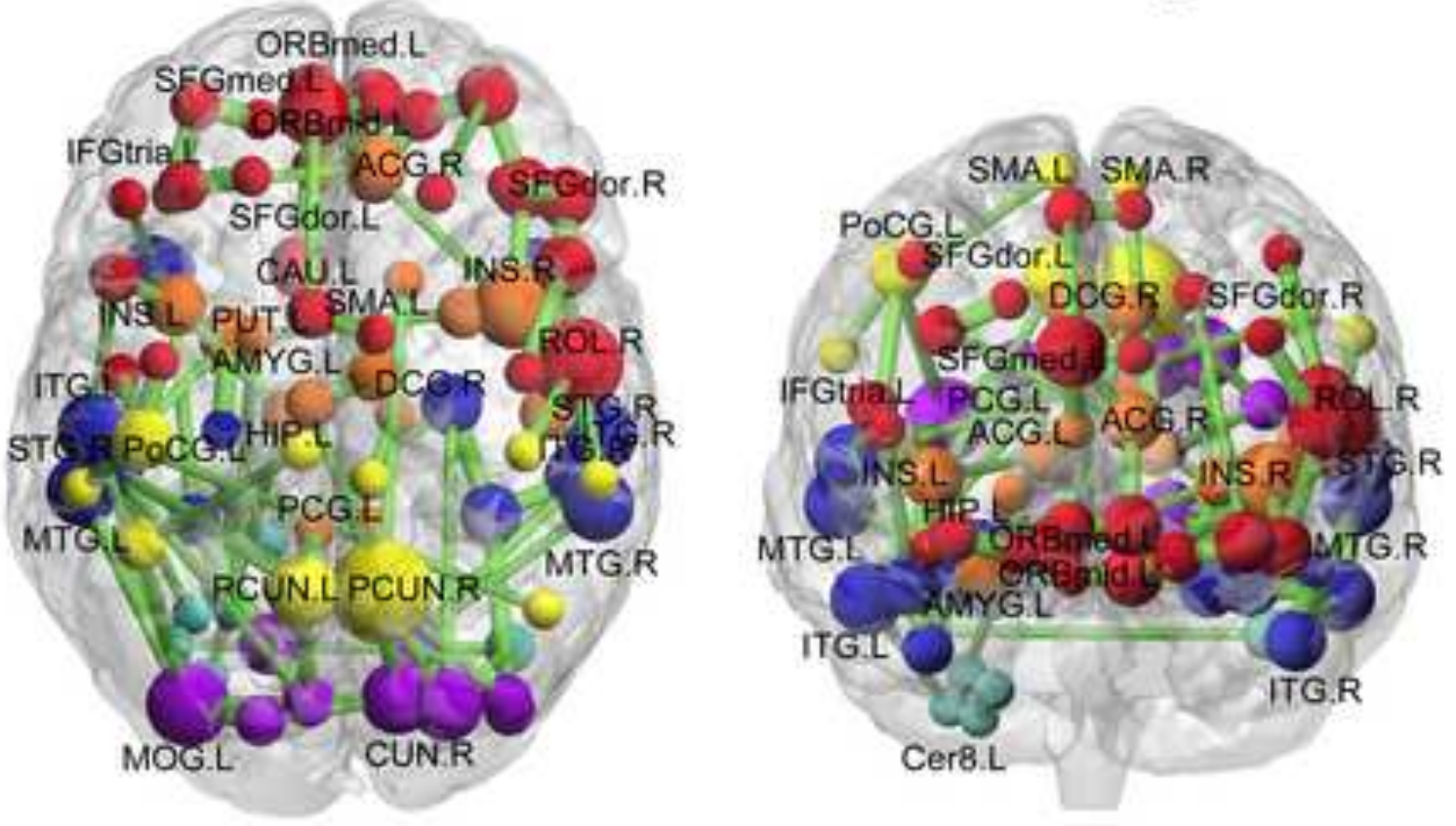

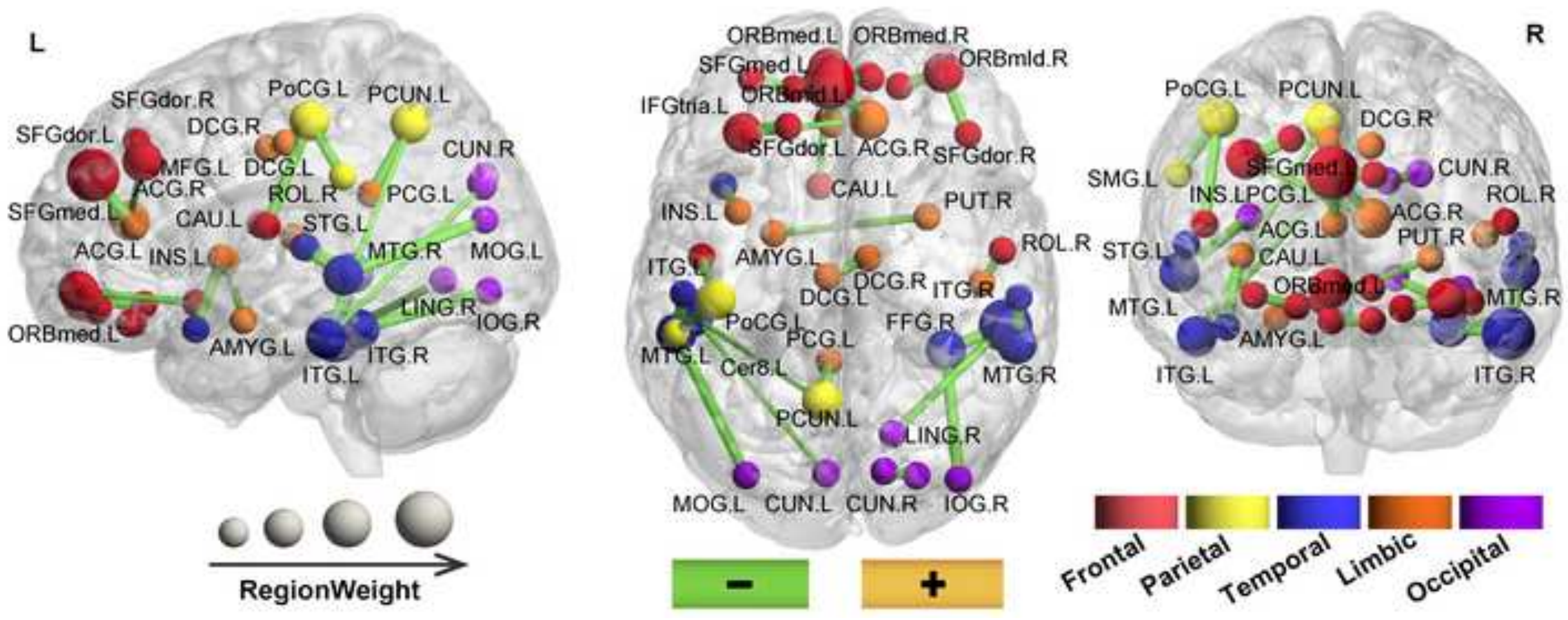